(2) Open Access Full Text Article

\title{
Prognostic significance of microRNA-100 in solid tumors: an updated meta-analysis
}

OncoTargets and Therapy

23 January 2017

Number of times this article has been viewed

\section{Jiangfeng Wang' \\ Miao $\mathrm{Yu}^{2}$ \\ Shanghui Guan' \\ Guangyu Zhang' \\ Jianbo Wang' \\ Yufeng Cheng'}

'Department of Radiation Oncology, Qilu Hospital of Shandong University, Jinan, People's Republic of China; ${ }^{2}$ School of Medicine, University of Washington, Seattle, WA, USA
Correspondence: Yufeng Cheng

Department of Radiation Oncology, Qilu Hospital of Shandong University, No 107

West Wenhua Road, Jinan 2500I2,

People's Republic of China

$\mathrm{Tel} / \mathrm{fax}+8653182169114$

Email qiluflk@163.com
Objective: The aim of this study was to identify prognostic significance of microRNA-100 (miR-100) in solid tumor.

Methods: Literature search was conducted in databases such as PubMed, Embase, and Web of Science, using the following words "(microRNA-100 OR miR-100 OR mir100) AND (tumor OR neoplasm OR cancer OR carcinoma OR malignancy)." The search was updated up until July 10, 2016. Newcastle-Ottawa scale was used to evaluate the quality of studies. Pooled hazard ratio (HR) with 95\% confidence interval (CI) for patients' survival was calculated by using a fixed-effects or a random-effects model on the basis of heterogeneity. Subgroup analysis, sensitive analysis, and meta-regression were used to investigate the sources of heterogeneity. Publication bias was evaluated by using Begg's and Egger's tests.

Results: A total of 16 articles with 1,501 patients were included in the present meta-analysis. It was demonstrated that a lower expression of miR-100 plays a negative role in the overall survival (OS) of patients with solid tumor ( $\mathrm{HR}=1.92$; 95\% $\mathrm{CI}=1.25-2.94)$. In addition, the association between miR-100 and prognosis was also revealed in the following subgroups: nonsmall-cell lung cancer (NSCLC; HR $=2.46 ; 95 \% \mathrm{CI}=1.98-3.06$ ), epithelial ovarian cancer ( $\mathrm{EOC}$; $\mathrm{HR}=2.29,95 \% \mathrm{CI}=1.72-3.04)$, and bladder cancer $(\mathrm{BC} ; \mathrm{HR}=4.14,95 \% \mathrm{CI}=1.85-9.27)$.

Conclusion: This meta-analysis indicates that lower expression of miR-100 is related to poorer OS in patients with solid tumor, especially in those with NSCLC, EOC, and BC. MiR-100 is a promising prognosis predictor and may be a potential target for therapy in the future.

Keywords: microRNA-100, prognosis, meta-analysis

\section{Introduction}

MicroRNAs, noncoding small RNAs, regulate mRNA at the posttranscriptional level, leading to degradation or inhibition of the target mRNA. Research on microRNAs began in 1993. ${ }^{1,2}$ Since then, an increasing number of microRNAs were discovered, and it was found that play an important role in a variety of biological activities including proliferation, differentiation, invasion, and apoptosis. ${ }^{3-5}$ The association between microRNAs and cancer was first reported in 2002 by Calin et al, who observed that miR-15 and miR-16 acted as negative regulators of Bcl-2 in cancer, especially chronic lymphocytic leukemia. ${ }^{6}$ It is now clear that microRNAs play a crucial role in cancer cells. Moreover, the clinical use of microRNA is being widely investigated. As a result, currently, multiple research studies have been exploring the prognostic function of microRNA-100 (miR-100) in cancer patients in order to find a reliable biomarker to guide for cancer treatment. ${ }^{7,8}$

As a member of the miR-99 family, miR-100 is located on Chromosome 11 at 11q24.1. Its aberrant expression was reported in various cancers including nasopharyngeal cancer (NPC), ${ }^{10}$ oral squamous cell carcinoma, ${ }^{11}$ esophageal adenocarcinoma (EA), ${ }^{12}$ 
small cell carcinoma of the cervix (SCCC), ${ }^{13}$ non-small-cell lung cancer (NSCLC), ${ }^{14,15}$ endometrioid endometrial carcinoma (EEC), ${ }^{16}$ epithelial ovarian cancer (EOC), ${ }^{17,18}$ bladder cancer (BC), ${ }^{19,20}$ renal cell carcinoma (RCC), ${ }^{21}$ esophageal squamous cell carcinoma (ESCC), ${ }^{22,23}$ hepatocellular carcinoma (HCC), ${ }^{24}$ colorectal cancer (CRC), ${ }^{25,26}$ and pancreatic adenocarcinoma. ${ }^{27}$ This aberrant expression of miR-100 not only has diagnostic implications but also can predict cancer patient survival. ${ }^{28}$ From this perspective, it may be feasible to predict prognosis based on miR-100 expression level. However, the correlation between them remains controversial to some extent. In some studies, miR-100 was reported as a tumor suppressor, whose low expression level was a negative prognostic factor. ${ }^{16,17}$ In contrast, in some articles, miR-100 was regarded as an oncogene..$^{21,27}$ Therefore, this metaanalysis was conducted to identify the relationship between miR-100 expression level and the survival of cancer patients, by pooling the hazard ratio (HR) from studies addressing the correlation between miR-100 and overall survival (OS) of patients with solid tumor.

\section{Materials and methods}

\section{Search strategy}

A literature search was conducted in databases such as PubMed, Embase, and Web of Science, using the following words "(microRNA-100 OR miR-100 OR mir100) AND (tumor OR neoplasm OR cancer OR carcinoma OR malignancy)." The search was updated up until July 10, 2016. In order not to miss the potentially related articles, reference lists were also screened.

\section{Inclusion and exclusion criteria}

Inclusion criteria were as follows: 1) studies exploring any of the following solid tumor (eg, head and neck, thyroid, non-small-cell or small cell lung, breast, esophageal, gastric, pancreatic, hepatobiliary, colorectal, anal, prostate, bladder, renal, cervical, endometrial and ovarian carcinoma, melanoma, and sarcomas); 2) studies dealing with miR-100 expression and OS; 3 ) studies that categorized patients into low- and high-expression groups based on the miR-100 expression; 4) studies providing HR directly or key information to calculate HR indirectly, such as Kaplan-Meier curves and original survival data; and 5) studies assessing miR-100 expression in tissue or blood. The following were the exclusion criteria: 1) studies on myelomas, lymphomas, or leukemia; 2) nonoriginal articles, such as reviews, articles, or letters; 3) laboratory studies on cell lines or animals level; 4) studies on a set of microRNAs but not miR-100 alone; and 5) studies on nondichotomous miR-100.

\section{Qualitative assessment}

Newcastle-Ottawa scale was used to evaluate the quality of the studies based on the following three aspects: selection, comparability, and outcome. ${ }^{29}$ A study can be given a maximum of one star for each item within the selection and outcome categories, and a maximum of two stars can be given for comparability category. The total stars that can be given for a study ranges from 0 to 9 . Studies with $\geq 6$ stars were considered as of high quality. ${ }^{30}$ Otherwise, studies were excluded from the final meta-analysis.

\section{Data extraction}

Data extraction was carried out by Wang and Cheng. The following details of each article were recorded: publication year, first author's last name, cancer type, treatment, sample size, stage of disease, miR-100 assay, the cutoff value to discriminate high or low expression of miR-100, sample sources, follow-up time, and HR (low versus high expression). HR $>1$ indicates that lower expression of miR-100 is an unfavorable prognostic factor, and HR $<1$ suggests that lower expression of miR-100 confers survival advantage to the patients. If the HR value was calculated by a multivariate analysis, then it was extracted directly; if multivariate analysis was not available, then results from univariate analysis were also accepted in the meta-analysis. If both multivariate analysis and univariate analysis were not offered, Kaplan-Meier curves were used to estimate HR value by using the described method. ${ }^{31}$ In addition, original survival data obtained, if possible, can also be used to calculate HR by conducting survival analysis.

\section{Statistical analysis}

In order to test the heterogeneity of pooled HR, Cochran's $Q$-test and Higgins $I^{2}$ statistics were performed. $P<0.05$ was considered statistically significant. Random-effects model was used to calculate pooled HR when between-study heterogeneity was revealed $(P<0.05)$, and fixed-effects model was conducted when between-study heterogeneity did not reach the statistical significance $(P>0.05)$. Subgroup analysis, sensitive analysis, and meta-regression were used to investigate the sources of heterogeneity. Publication bias was assessed by using Begg's test and Egger's test. ${ }^{32,33}$ STATA version 12.0 (Stata Corporation, College Station, TX, USA) was used to perform all the analyses.

\section{Results \\ Study selection}

In total, 179, 482, and 292 records were identified from PubMed, Embase, and Web of Science, respectively 
(Figure 1). After excluding the replicate records, 272 articles were left. Reviewers Wang and Huang carefully read the abstract or full text as required and removed 658 records because they were review articles, laboratory studies, and so on. Then, 23 publications were retrieved for further analysis; of these studies, two studies focused on biochemical-free survival and recurrence-free survival of prostate cancer instead of OS $;^{34,35}$ three articles did not address solid tumor. ${ }^{36-38}$ In addition, a study by Wang et al used malignant effusion, rather than tissue or blood, to assess miR-100 expression in NSCLC patients. ${ }^{39}$ Therefore, according to the inclusion and exclusion criteria in the present study, 17 articles were accepted for Newcastle-Ottawa scale.

\section{Qualitative assessment}

Results from Newcastle-Ottawa scale demonstrated that two studies received eight stars, eight articles received seven stars, six articles received six stars, and one article received one star. Thus, a study by Zhang et al was excluded as it was a low-quality article (Table 1$).{ }^{40}$

\section{Summary of the analyzed studies}

After filtering, 16 studies published between 2011 and 2016 were considered for the final meta-analysis (Table 2).
The cancer types included EA, SCCC, EEC, human EOC, $\mathrm{BC}$, NSCLC, RCC, ESCC, HCC, CRC, and pancreatic ductal adenocarcinoma. Only four studies clearly stated the research-related treatment. As revealed in Table 2, most studies (11 of 16) did not clarify whether patient received adjuvant therapy after surgery. Study sample sizes ranged from 42 to 172 with a total of 1,501 patients from the People's Republic of China, the USA, Poland, Iran, and Germany. Nine studies enrolled patients with stages I-IV, whereas a study by Dhayat only explored miR-100 in patients with Stage II pancreatic ductal adenocarcinoma. ${ }^{27}$ Four studies did not specify the stage of disease in the study population. Quantitative real-time polymerase chain reaction (qRT-PCR) was used to assess miR-100 expression in all the studies, and the cutoff value varied among studies with median expression of miR-100 the most widely used. All the authors used tissues as the source of miR-100. The majority of HRs (13 of 16) were reported in the present analysis, all of which were calculated by using a multivariate analysis, whereas four HRs were estimated by analyzing Kaplan-Meier curves.

\section{Meta-analysis results}

Due to obvious heterogeneity $\left(I^{2}=85.9 \% ; P<0.001\right)$, random-effects model was used to calculate pooled HR

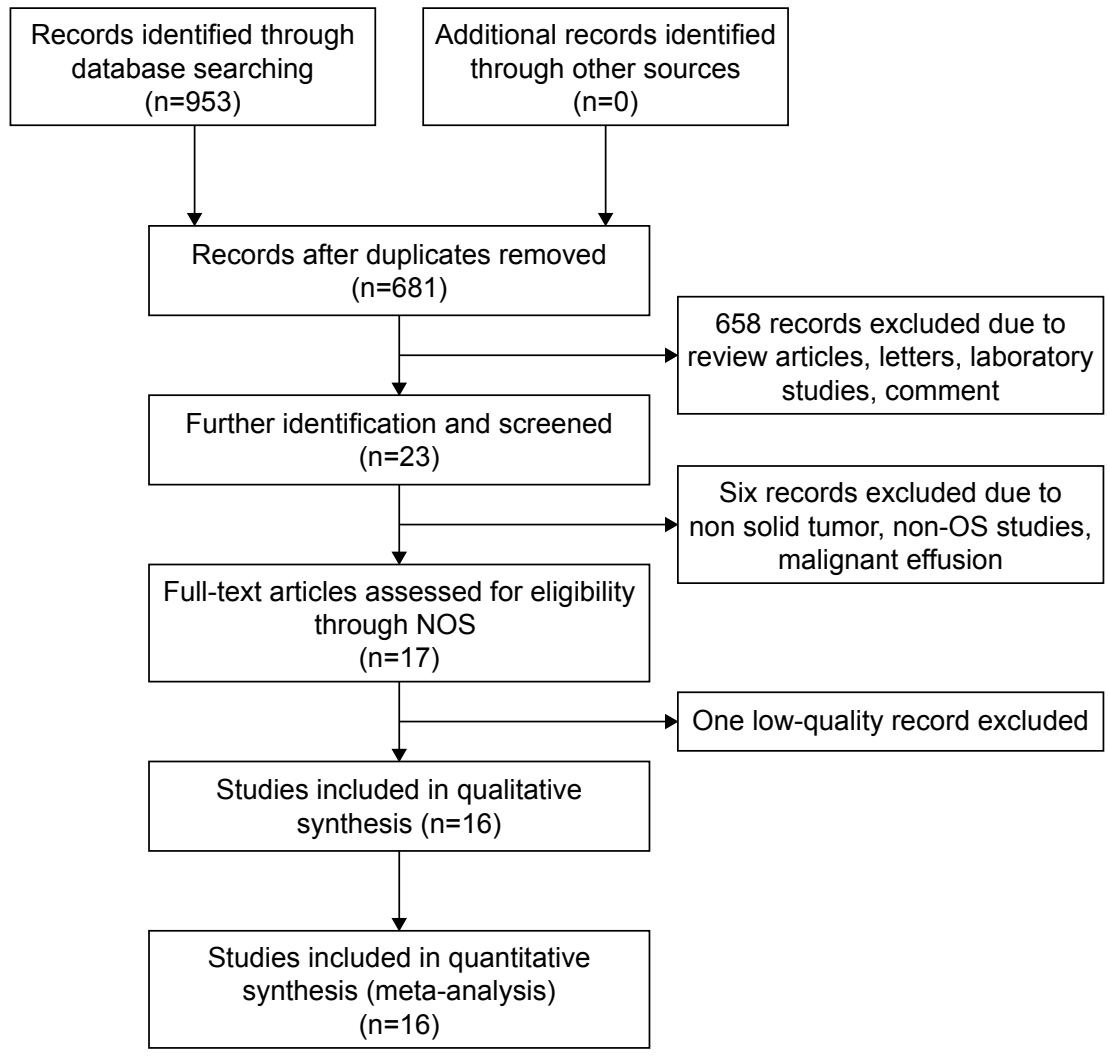

Figure I Flow diagram of filtering studies.

Abbreviations: NOS, Newcastle-Ottawa scale; OS, overall survival. 
Table I Newcastle-Ottawa scale

\begin{tabular}{|c|c|c|c|c|}
\hline & Selection & Comparability & Outcome & Total \\
\hline Feber et $\mathrm{al}^{12}$ & 4 & $0^{e, f}$ & $2^{g}$ & 6 \\
\hline Huang et $\mathrm{al}^{13}$ & 4 & $0^{e, f}$ & $2^{g}$ & 6 \\
\hline Torres et $\mathrm{al}^{16}$ & 4 & $0^{e, f}$ & $2^{g}$ & 6 \\
\hline Peng et $\mathrm{al}^{17}$ & 4 & $\mathrm{I}^{\mathrm{e}}$ & $2^{g}$ & 7 \\
\hline Wang et al $^{19}$ & 4 & $\mathrm{l}^{\mathrm{e}}$ & $2^{g}$ & 7 \\
\hline Liu et $\mathrm{al}^{14}$ & 4 & $\mathrm{I}^{\mathrm{e}}$ & $2^{g}$ & 7 \\
\hline Wang et $\mathrm{al}^{2 !}$ & 4 & $\mathrm{I}^{\mathrm{e}}$ & $2^{g}$ & 7 \\
\hline Sun et $\mathrm{al}^{22}$ & 4 & 2 & $\left.\right|^{g, h}$ & 7 \\
\hline Chen et $\mathrm{al}^{24}$ & 4 & $\mathrm{I}^{\mathrm{e}}$ & $2^{g}$ & 7 \\
\hline Chen et $\mathrm{al}^{25}$ & 4 & $\mathrm{I}^{\mathrm{e}}$ & $2^{g}$ & 7 \\
\hline Zhou et $\mathrm{al}^{23}$ & 4 & $\mathrm{I}^{\mathrm{e}}$ & 3 & 8 \\
\hline Cao et $\mathrm{al}^{20}$ & 4 & $\mathrm{I}^{\mathrm{e}}$ & $2^{g}$ & 7 \\
\hline Zhang et $\mathrm{al}^{40}$ & $0^{\mathrm{a}-\mathrm{d}}$ & $0^{e, f}$ & g,h & I \\
\hline Luo et al ${ }^{15}$ & 4 & $0^{e, f}$ & $2^{g}$ & 6 \\
\hline Dhayat et $\mathrm{al}^{27}$ & 4 & $I^{f}$ & 3 & 8 \\
\hline Zhang et $\mathrm{al}^{26}$ & 4 & $\mathrm{I}^{\mathrm{e}}$ & $\left.\right|^{g, i}$ & 6 \\
\hline Azizmohammadi et al $^{18}$ & 4 & $\mathrm{I}^{\mathrm{e}}$ & $\left.\right|^{g, h}$ & 6 \\
\hline
\end{tabular}

Notes: Reasons for lost stars: ano description of the derivation of the cohort; ${ }^{b}$ no description of the derivation of the nonexposed cohort; ' no description of exposure ascertainment; ${ }^{d}$ no description of whether outcome of interest was not present at the start of study; 'estudy not controlling the most important factor such as TNM stage; 'study not controlling other additional factors, such as age, gender, and smoke; gno description of outcome assessment; hinadequacy of follow-up of cohorts; 'followup not long enough for outcomes to occur.

(HR $=1.92 ; 95 \%$ confidence interval $[\mathrm{CI}]=1.25-2.94)$, suggesting that lower expression level of miR-100 significantly predicted poorer OS in patients with solid tumor (Figure 2).

In order to identify the potential source of heterogeneity, subgroups analysis was conducted based on the patient origin, cancer type, and the methods used to calculate HR (Table 3). The association between miR-100 and OS was also revealed in the following subgroups: $\mathrm{NSCLC}(\mathrm{HR}=2.46 ; 95 \%$ $\mathrm{CI}=1.98-3.06$; fixed-effects model), EOC (HR $=2.29 ; 95 \%$ $\mathrm{CI}=1.72-3.04$; fixed-effects model), and $\mathrm{BC}$ ( $\mathrm{HR}=4.14$; $95 \%$ CI $=1.85-9.27$; fixed-effects model). Notably, significant heterogeneity was not found among these studies, with $P$-value of heterogeneity being $0.696,0.07$, and 0.146 , respectively. However, no statistic difference was reported in esophageal carcinoma (HR $=1.82 ; 95 \% \mathrm{CI}=0.32-10.32$; randomeffects model) and CRC (HR $=1.70 ; 95 \% \mathrm{CI}=0.93-3.09$; random-effects model). The prognostic role of miR-100 was also significant in the following subgroups: HR by report or multivariate analysis ( $\mathrm{HR}=1.91 ; 95 \% \mathrm{CI}=1.91-3.06$; random-effects model) and Asian patients ( $\mathrm{HR}=2.38 ; 95 \%$ CI $=1.56-3.66$; random-effects model).

Sensitivity analysis was conducted by sequentially omitting individual studies, indicating that there was not a single study that significantly contributed to heterogeneity (Figure 3). Furthermore, a meta-regression was also conducted to explore the potential factors that are responsible for heterogeneity. The results showed that the following factors could partly explain the heterogeneity but did not reach statistical significance: patient origin $\left(I^{2}=85.68 \%\right.$; adjusted $\left.R^{2}=16.54 \% ; P=0.06\right)$, cancer type $\left(I^{2}=83.65 \%\right.$; adjusted $\left.R^{2}=10.95 \% ; P=0.59\right)$, and multivariate analysis $\left(I^{2}=86.84 \%\right.$, adjusted $R^{2}=-8.60 \% ; P=0.97$ ).

Publication bias was evaluated by using Begg's funnel plot and Egger's test. Although the funnel plot revealed slight publication bias, $P$-values of Begg's and Egger's tests were 0.753 and 0.056 , respectively, showing no evidence for significant publication bias (Figure 4).

\section{Discussion}

Lack of a reliable biomarker to predict prognosis is a main hurdle to realize individualized treatment. Fortunately, emerging data favor the potential use of miR-100 as a cancer prognostic predictor. In recent years, downstream targets of miR-100 have widely been investigated in several signal pathways, which may provide evidence for its potential clinical use at a molecule level. First, miR-100 facilitated cancer cell proliferation and growth by targeting the mammalian target of rapamycin (mTOR) and fibroblast growth factor receptors (FGFRs). By using GeneChip ${ }^{\circledR}$ miRNA Array and qRT-PCR, Xu et al ${ }^{41}$ identified decreased expression of miR-100 in bladder tumor tissues and found that ectopic restoration of miR-100 expression suppressed tumor cell proliferation. Furthermore, bioinformatic analysis indicated that mTOR was the direct target of miR-100. ${ }^{41}$ Consistently, another study on esophageal squamous cell cancer also supported this report. Sun et al transfected esophageal squamous cell cancer cell lines with miR-100 precursor molecules and reported the subsequent inhibition of cell proliferation, which was attributed to the fact that miR-100 downregulated the expression of mTOR by directly targeting its 3 'UTR in a posttranscriptional manner. ${ }^{22}$ In gynecologic cancer, this interaction exists. Torres et al observed that decreased miR-100 in EEC tissues coexisted with increased mTOR kinase. ${ }^{16}$ With respect to cell growth, another potential target of miR-100 may be FGFRs. Bi et al transfected U2OS cells (human osteosarcoma line) with a miR-100 construct or with an antisense of miR-100. ${ }^{42}$ It was observed that overexpression of miR-100 led to decreased expression of FGFR3 protein. Conversely, inhibition of miR-100 induced FGFR3 protein expression. Bioinformatics analysis and luciferase reporter assay implied that $3^{\prime}$ UTR of FGFR3 mRNA was the direct target of miR-100. Based on these experimental results, $\mathrm{Bi}$ et al ${ }^{42}$ pointed out that miR-100 served as a tumor suppressor by 


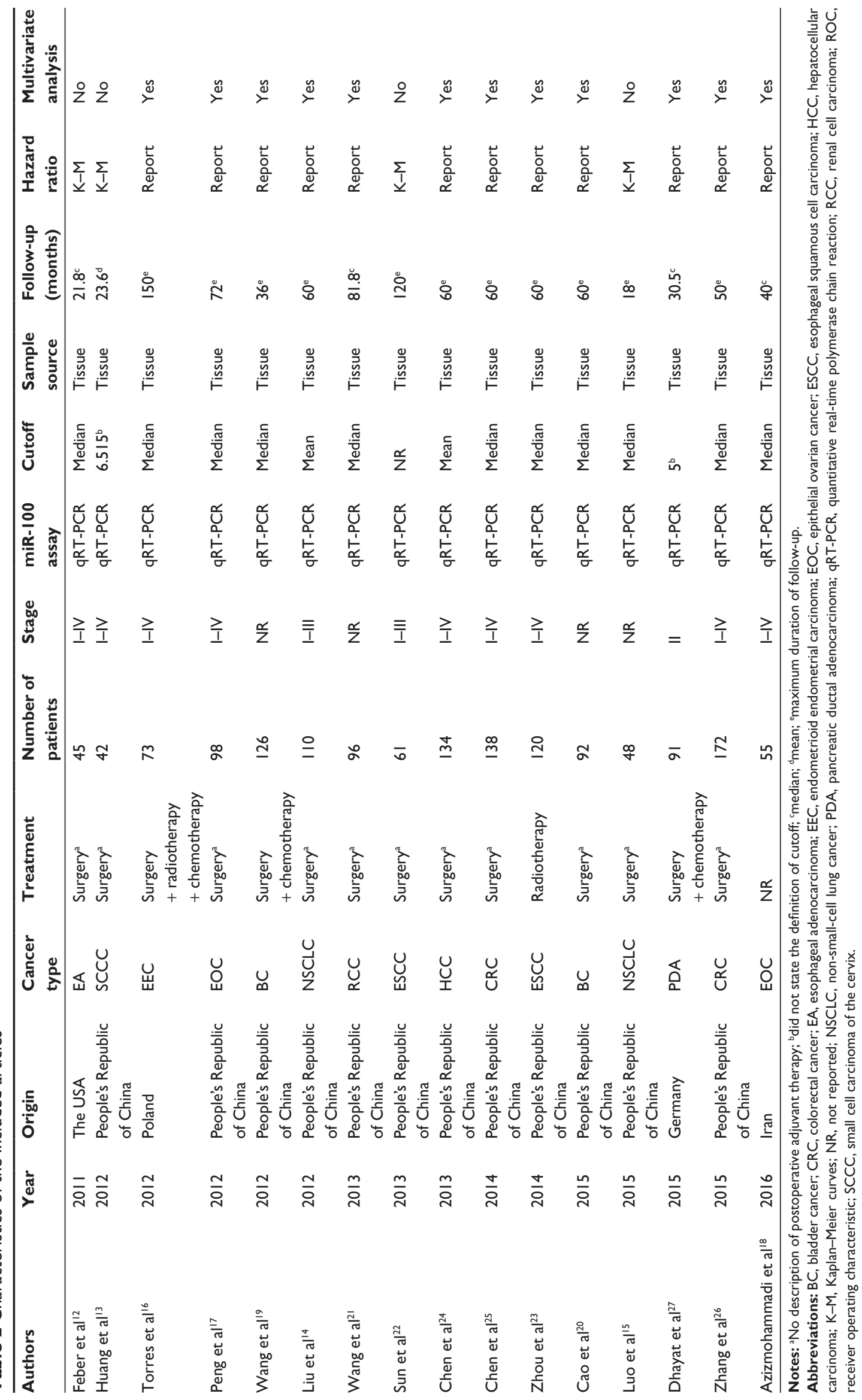




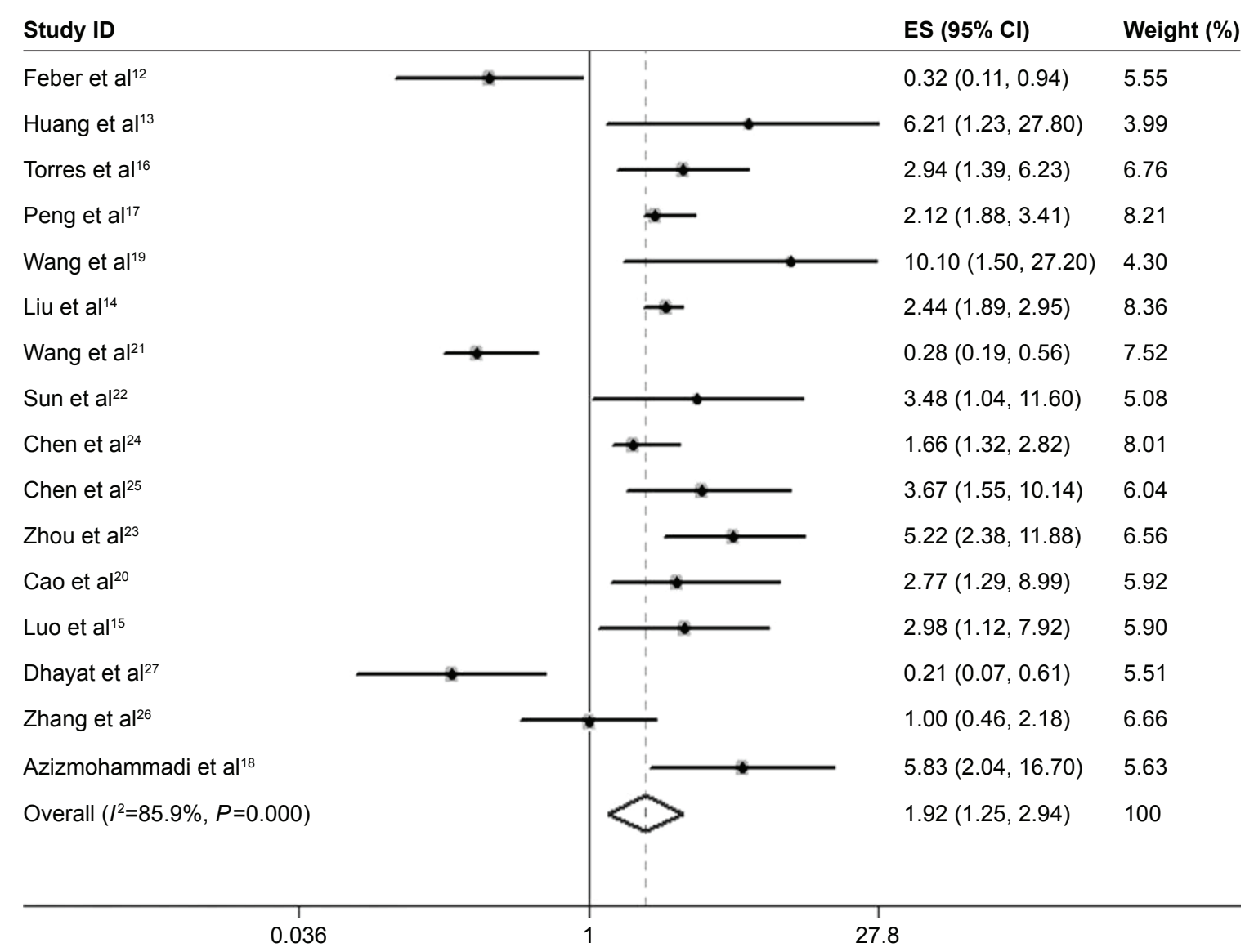

Figure 2 Forest plot of the relationship between miR-100 and overall survival in solid tumor.

Note: Weights are from random-effects analysis.

Abbreviations: $\mathrm{Cl}$, confidence interval; $\mathrm{ES}$, effect size.

preventing translation of FGFR3s. Another study on BC also revealed that the reduction of miR-100 expression induced sixfold upregulation of FGFR3 protein through an interaction with FGFR3 mRNA. ${ }^{43}$ Similar result was also reported in non-small-cell lung. ${ }^{15}$ Second, miR-100 participated in cancer cell apoptosis, which also determines the development of the tumor. Chen et al observed that miR-100 precursor elevated expression of cleaved caspase- 3 and pro-caspase- 3 protein, which are two key apoptosis proteins. ${ }^{24}$ In order to find the mechanism, flow cytometry and Western blot analysis were used to assess the apoptosis in siRNA/plk 1-transfected human hepatocellular cells. The outcome showed that

Table 3 Subgroup analysis

\begin{tabular}{|c|c|c|c|c|}
\hline \multirow[t]{2}{*}{ Subgroup } & \multicolumn{2}{|c|}{ Heterogeneity } & \multirow[t]{2}{*}{ HR (95\% Cl) } & \multirow[t]{2}{*}{$P$-value } \\
\hline & $I^{2}$ & $P$-value & & \\
\hline \multicolumn{5}{|l|}{ Patient origin } \\
\hline Asian & $84.6 \%$ & $<0.001$ & $2.38(1.55-3.66)^{\mathrm{a}}$ & $<0.001$ \\
\hline Non-Asian & $90.0 \%$ & $<0.001$ & $0.60(0.10-3.50)^{\mathrm{a}}$ & 0.574 \\
\hline \multicolumn{5}{|l|}{ Cancer type } \\
\hline Non-small-cell lung cancer & 0 & 0.696 & $2.46(1.98-3.06)^{\mathrm{b}}$ & $<0.001$ \\
\hline Epithelial ovarian cancer & $69.6 \%$ & 0.070 & $2.29(1.72-3.04)^{b}$ & $<0.001$ \\
\hline Bladder cancer & $52.7 \%$ & 0.146 & $4.14(1.85-9.27)^{b}$ & 0.001 \\
\hline Esophageal cancer & $88.5 \%$ & $<0.001$ & I. $82(0.32-10.32)^{\mathrm{a}}$ & 0.497 \\
\hline Colorectal cancer & $77.1 \%$ & 0.037 & $1.7(0.93-3.09)^{\mathrm{b}}$ & 0.084 \\
\hline \multicolumn{5}{|l|}{ Multivariate analysis } \\
\hline Yes & $88.0 \%$ & $<0.001$ & $1.91(1.19-3.06)^{\mathrm{a}}$ & 0.007 \\
\hline No & $79.4 \%$ & 0.002 & $2.03(0.55-7.42)^{\mathrm{a}}$ & 0.286 \\
\hline
\end{tabular}

Notes: ${ }^{R}$ Random-effects model; bfixed-effects model.

Abbreviations: $\mathrm{Cl}$, confidence interval; $\mathrm{HR}$, hazard ratio. 


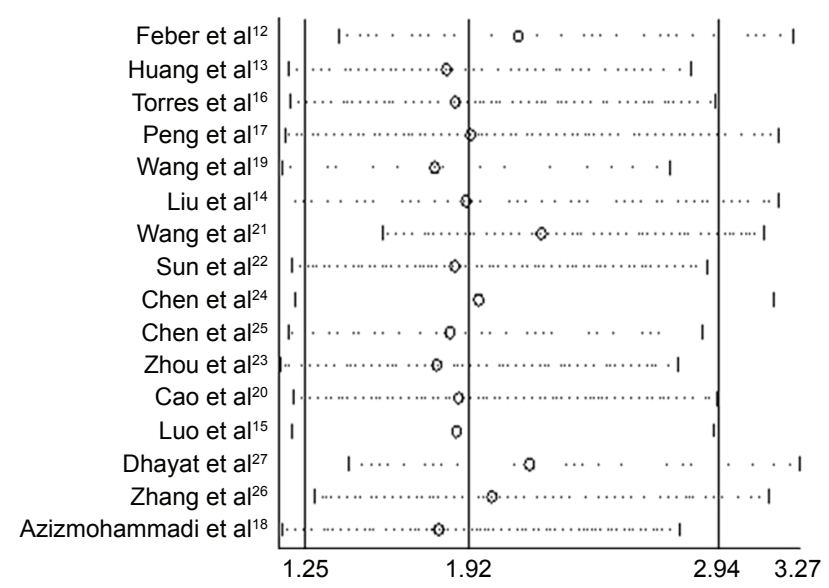

Figure 3 Sensitivity analysis: meta-analysis of random-effects estimates (exponential form) with studies omitted.

siRNA/plk 1 could mimic the effect of miR-100, indicating plk1 as a potential target of miR-100. In another digestive cancer, gastric cancer, Notch pathway was reported to participate in the miR-100-mediated apoptosis, which is initiated by the interaction between miR-100 and HS3ST2. ${ }^{44}$ Third, miR100 regulated several components of $\mathrm{Wnt} / \beta$-catenin pathway, including $\beta$-catenin, metalloproteinase-7, T-cell factor-4, and lymphoid enhancing factor-1, which were closely related to cell invasion and tumor metastasis. ${ }^{45}$ Transwell migration and invasion assay by Jiang showed that transfection of miR-100 mimic suppressed migration and invasion of breast cancer compared to the control group. The direct target of miR-100 in Wnt/ $\beta$-catenin pathway may be FZD-8. ${ }^{46}$ Bhushan et al also found that downregulation of miR-100 may account for less invasion of breast cancer cells. ${ }^{47}$ In addition, $\mathrm{Fu}$ et al also consolidated the role miR-100 played in invasion and metastasis of esophageal squamous cell cancer by using

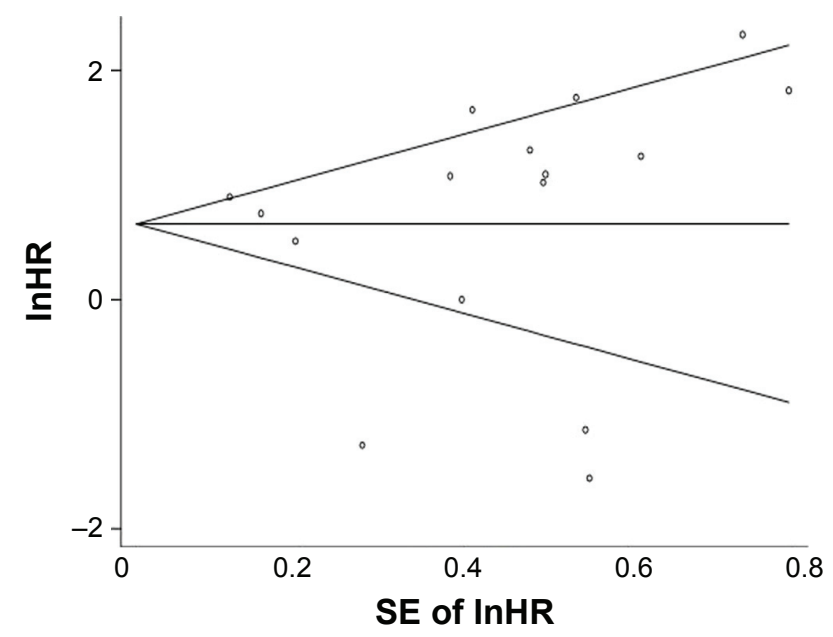

Figure 4 Publication bias: Begg's funnel plot with pseudo 95\% confidence limits. Abbreviations: HR, hazard ratio; SE, standard error. bioinformatics analyses. ${ }^{48}$ Fourth, cell cycle arrest, which is responsible for radio-resistance or chemo-resistance, was mediated by miR-100. Xiao et al discovered that the introduction of miR-100 mimic in H69 cells (human small cell lung cancer cell line) led to downregulation of HOXA1 protein, inducing G0-G1 and G1-M cell cycle arrest, which was associated with drug resistance. ${ }^{49} \mathrm{~A}$ study by Shi et al documented that miR-100 can target plk1 and mediated G2/M arrest in NPC. ${ }^{10}$ Together, miR-100 might be a promising prognostic predictor.

Despite the sort of inconsistency, clinical investigations favor predictive use of miR-100 for the survival of cancer patients. Peng et al retrospectively collected fresh surgical tissues from 98 patients with EOC and used qRT-PCR assay to assess the expression level of miR-100. ${ }^{17}$ It is revealed that the expression of miR-100 was significantly lower in epithelial ovarian tumor tissue than in adjacent normal tissue. Multivariate analysis demonstrated that lower expression of miR-100 was accompanied by shorter survival ( $\mathrm{HR}=2.12$; 95\% CI $=1.88-3.41$ ). Further evidence on endometrial carcinoma also supported the finding that decreased expression level of miR-100 worsens prognosis, with a HR of 2.94 (low/ high). ${ }^{16}$ Similar results were obtained for digestive system cancers. Zhou investigated 120 patients with esophageal squamous cell cancer who received radiotherapy alone and concluded that HRs for locoregional progression-free survival (PFS), distant PFS, and OS (low/high) were 8.9, 8.34 , and 8.02, respectively, suggesting that downregulation of miR-100 indicated poor prognosis. ${ }^{23}$ In BC, Wang et al reported that 3-year OS and 3-year PFS for high expression level of miR-100 were 33 and 26 months, respectively; on the other hand, for low expression level of miR-100, they were merely 23 and 16 months, respectively. ${ }^{19}$ However, it should be noted that literature on the prognostic value of miR-100 reveals conflicting findings. Wang et al found increased miR-100 expression in cancer tissues compared with normal tissues in patients with renal cell cancer. ${ }^{21} \mathrm{HRs}$ for OS and tumor-specific survival were 0.28 and 0.41 , respectively, both of which were statistically significant, suggesting that lower expression of miR-100 confers survival advantage. Dhayat et al focused on pancreatic ductal adenocarcinoma Union for International Cancer Control stage II and found that lower level of miR-100 is a favorable factor to predict treatment response and OS. ${ }^{27}$ Apart from studies revealing the survival difference, a study by Zhang showed that the difference in miR-100 expression level did not lead to statistic difference in the survival of patients with CRC. ${ }^{26}$ In order to clarify the relationship between miR-100 and survival of patients with solid tumor, this meta-analysis was conducted. 
In order to ensure reliable outcome, the inclusion criteria were strictly limited. First, source of RNA was restricted to tissue or blood. As a result, a study by Wang et al was not accepted, because it explored miR-100 in malignant effusion in NSCLC. ${ }^{39}$ Second, Newcastle-Ottawa scale was used. As shown in Table 1, most studies (16 of 17) were evaluated as of high quality; however, only two studies received nine stars, the highest score. The most common reasons why the research was deprived of stars lay in the comparability and outcome aspects. In terms of comparability, majority of the studies (15 of 17) did not control the most important factor such as TNM stage; some studies (6 of 17) did not control other less important factors, such as age, gender, and smoking status of the patients, which would bring potential bias to the survival outcome. In addition, it is necessary for prognosis studies to describe whether the survival outcome was attained by self-report or blind assessment, which was stated in Newcastle-Ottawa scale. However, only two studies mentioned this category. ${ }^{23,27}$ Lastly, follow-up rate is not fully recorded. Only a minority of studies described the number of lost patients and offered the reason for loss. Considering these reasons, more stars have been provided in outcome category. It is noteworthy that Zhang et al analyzed survival data from the TCGA database and did not provide sufficient related information to get at least six stars according to Newcastle-Ottawa scale. ${ }^{40}$ Hence, although it investigated the association between miR-100 expression and OS, it was excluded as a study of low quality.

Finally, 16 studies were included. According to the present study, decreased miR-100 expression level confers survival disadvantage in patients with solid tumor ( $\mathrm{HR}=1.92 ; 95 \% \mathrm{CI}=1.25-2.94)$. This relationship was also found in the following subgroups: NSCLC (HR $=2.46 ; 95 \%$ $\mathrm{CI}=1.98-3.06$; fixed-effects model), EOC ( $\mathrm{HR}=2.29 ; 95 \%$ $\mathrm{CI}=1.72-3.04$; fixed-effects model), and $\mathrm{BC}$ ( $\mathrm{HR}=4.14$; $95 \%$ CI $=1.85-9.27$; fixed-effects model). In order to find the potential source of heterogeneity, first, subgroup analysis was conducted, which revealed that heterogeneity declined dramatically in NSCLC group $\left(I^{2}=0 ; P=0.70\right)$, EOC group $\left(I^{2}=69.6 \% ; P=0.07\right)$, and $\mathrm{BC}$ group $\left(I^{2}=52.7 \%\right.$; $P=0.15)$. Therefore, cancer type might partly account for heterogeneity. Previously, several meta-analyses classified subgroups according to the method used to assess RNA (qRT-PCR versus in situ hybridization) and the source of RNA (blood versus tissue). ${ }^{30}$ Nonetheless, these variants were similar between the studies included in the present meta-analysis. As a result, the possibility of these variants as the source of heterogeneity was eliminated, and there was no requirement to perform further subgroup analysis. Owing to the results that sensitivity analysis did not reveal the source of heterogeneity, meta-regression was further conducted, which revealed that $P$-value of patient origin, the method used to calculate HR, and cancer type were all $>0.05$, with adjusted $R^{2}$ being $16.54 \%,-8.6 \%$, and $-10.95 \%$, respectively. Meta-regression results showed that although these factors might explain part of heterogeneity, but it did not reach statistical significance. Meta-regression could have been used to analyze whether other factors such as follow-up time, related treatment methods, and cutoff value had an impact on heterogeneity, but, due to insufficient information on or remarkably different definition of cutoff value, the necessity and scientific implication to carry out this metaregression in these variants were uncertain.

As it is well known, meta-analysis results are more robust than the result of single research. However, it is noteworthy that strict inclusion and exclusion criteria are a prerequisite for pooled data, which made the present results more reliable than the previous ones. ${ }^{50,51}$ Despite careful assessment of study quality, there are limitations in the present study. First, heterogeneity among the studies included is relatively high. According to subgroup analysis, tumor type could partly explain the source of heterogeneity. Nonetheless, other potential factors may also exist. For instance, pathological type may be a potential source. The pooled HR from ESCC subgroup was $4.61\left(95 \% \mathrm{CI}=2.36-8.99 ; I^{2}=0\right.$; fixed-effects model) compared to esophageal cancer ( $\mathrm{HR}=1.82 ; 95 \%$ CI $=0.32-10.32 ; I^{2}=88.5 \%$; random-effects model). ${ }^{22,23}$ In addition, because of insufficient data, it was difficult to further assess the effects of other variants on heterogeneity. For instance, TNM stage, postoperative treatment, and follow-up method were not clearly stated in all the studies. Second, the power of miR-100 as a stand-alone biomarker is presumably weak and varies for different tumor types, given the fact that interaction between microRNA and its target is complicated. Undoubtedly, microRNA signature may be a better option that has been investigated in other microRNAs. ${ }^{52}$ However, to date, such microRNA signature comprising miR-100 was not reported. Third, the definition of cutoff value varied among the studies. Some research studies chose median value to define the expression level of miR-100, yet some used mean value or value calculated by receiver operating characteristic curve. Finally, although publication bias was not revealed by Begg's and Egger's tests, the funnel plot is slightly asymmetric. The limitations emphasize the need for well-designed and well-conducted prognostic studies on miR-100 in the future. 


\section{Conclusion}

This meta-analysis indicated that lower expression level of miR-100 is related to poorer OS in solid tumor, especially in patients with NSCLC, EOC, and BC.

\section{Acknowledgments}

This work was supported by Youth Science Found of Qilu Hospital of Shandong University, Science and Technology plan project of Shandong province (2012GSF11852) and National Natural Science Foundation of China (81572958).

\section{Disclosure}

The authors report no conflicts of interest in this work.

\section{References}

1. Lee RC, Feinbaum RL, Ambros V. The C. elegans heterochronic gene lin-4 encodes small RNAs with antisense complementarity to lin-14. Cell. 1993;75(5):843-854.

2. Wightman B, Ha I, Ruvkun G. Posttranscriptional regulation of the heterochronic gene lin-14 by lin-4 mediates temporal pattern formation in C. elegans. Cell. 1993;75(5):855-862.

3. Yoshimura A, Numakawa T, Odaka H, Adachi N, Tamai Y, Kunugi H. Negative regulation of microRNA-132 in expression of synaptic proteins in neuronal differentiation of embryonic neural stem cells Neurochem Int. 2016;97:26-33.

4. Guan B, Li Q, Shen L, et al. MicroRNA-205 directly targets Kruppellike factor 12 and is involved in invasion and apoptosis in basal-like breast carcinoma. Int J Oncol. 2016;49(2):720-734

5. Zhang Y, Xue C, Zhu X, Zhu X, Xian H, Huang Z. Suppression of microRNA-125a-5p upregulates the TAZ-EGFR signaling pathway and promotes retinoblastoma proliferation. Cell Signal. 2016; 28(8):850-860.

6. Calin GA, Dumitru CD, Shimizu M, et al. Frequent deletions and down-regulation of micro-RNA genes miR15 and miR16 at 13q14 in chronic lymphocytic leukemia. Proc Natl Acad Sci US A. 2002;99(24): 15524-15529.

7. Wang W, Li J, Zhu W, et al. MicroRNA-21 and the clinical outcomes of various carcinomas: a systematic review and meta-analysis. $B M C$ Cancer. 2014; 14:819.

8. Dai SL, Zhou J, Pan C, et al. Prognostic value of microRNA-145 in patients with various cancers: a meta-analysis. Cancer Biomark. 2015;15(4): 507-513.

9. Qin C, Huang RY, Wang ZX. Potential role of miR-100 in cancer diagnosis, prognosis, and therapy. Tumour Biol. 2015;36(3):1403-1409.

10. Shi W, Alajez NM, Bastianutto C, et al. Significance of Plk1 regulation by miR-100 in human nasopharyngeal cancer. Int J Cancer. 2010; 126(9):2036-2048.

11. Henson BJ, Bhattacharjee S, O'Dee DM, Feingold E, Gollin SM. Decreased expression of miR-125b and miR-100 in oral cancer cells contributes to malignancy. Genes Chromosomes Cancer. 2009;48(7): 569-582.

12. Feber A, Xi L, Pennathur A, et al. MicroRNA prognostic signature for nodal metastases and survival in esophageal adenocarcinoma. Ann Thorac Surg. 2011;91(5):1523-1530.

13. Huang L, Lin JX, Yu YH, Zhang MY, Wang HY, Zheng M. Downregulation of six microRNAs is associated with advanced stage, lymph node metastasis and poor prognosis in small cell carcinoma of the cervix. PLoS One. 2012;7(3):e33762.

14. Liu J, Lu KH, Liu ZL, Sun M, De W, Wang ZX. MicroRNA-100 is a potential molecular marker of non-small cell lung cancer and functions as a tumor suppressor by targeting polo-like kinase 1. BMC Cancer. $2012 ; 12: 519$
15. Luo J, Chen B, Ji XX, Zhou SW, Zheng D. Overexpression of miR100 inhibits cancer growth, migration, and chemosensitivity in human NSCLC cells through fibroblast growth factor receptor 3. Tumour Biol. Epub 2015 Aug 28.

16. Torres A, Torres K, Pesci A, et al. Deregulation of miR-100, miR-99a and miR-199b in tissues and plasma coexists with increased expression of mTOR kinase in endometrioid endometrial carcinoma. BMC Cancer. 2012;12:369.

17. Peng DX, Luo M, Qiu LW, He YL, Wang XF. Prognostic implications of microRNA-100 and its functional roles in human epithelial ovarian cancer. Oncol Rep. 2012;27(4):1238-1244.

18. Azizmohammadi S, Azizmohammadi S, Safari A, et al. The role and expression of miR-100 and miR-203 profile as prognostic markers in epithelial ovarian cancer. Am J Transl Res. 2016;8(5):2403-2410.

19. Wang S, Xue S, Dai Y, et al. Reduced expression of microRNA-100 confers unfavorable prognosis in patients with bladder cancer. Diagn Pathol. 2012;7:159.

20. Cao YH, Zhang HH, Xu HF, Duan YJ, Li Q, Huang B. Prognostic role of microRNA-100 in patients with bladder cancer. Genet Mol Res. 2015; 14(4):15948-15954.

21. Wang G, Chen L, Meng J, Chen M, Zhuang L, Zhang L. Overexpression of microRNA-100 predicts an unfavorable prognosis in renal cell carcinoma. Int Urol Nephrol. 2013;45(2):373-379.

22. Sun J, Chen Z, Tan X, et al. MicroRNA-99a/100 promotes apoptosis by targeting mTOR in human esophageal squamous cell carcinoma. Med Oncol. 2013;30(1):411.

23. Zhou S, Yang B, Zhao Y, Xu S, Zhang H, Li Z. Prognostic value of microRNA-100 in esophageal squamous cell carcinoma. J Surg Res. 2014;192(2):515-520.

24. Chen P, Zhao X, Ma L. Downregulation of microRNA-100 correlates with tumor progression and poor prognosis in hepatocellular carcinoma Mol Cell Biochem. 2013;383(1-2):49-58.

25. Chen P, Xi Q, Wang Q, Wei P. Downregulation of microRNA-100 correlates with tumor progression and poor prognosis in colorectal cancer. Med Oncol. 2014;31(10):235.

26. Zhang S, Yuan W, Tang W, Xu C, Ma J. [Expression of microRNA-100 and its relation with prognosis of colorectal cancer]. Zhonghua Zhong Liu Za Zhi. 2015;37(8):603-608. Chinese.

27. Dhayat SA, Abdeen B, Kohler G, Senninger N, Haier J, Mardin WA. MicroRNA-100 and microRNA-21 as markers of survival and chemotherapy response in pancreatic ductal adenocarcinoma UICC stage II. Clin Epigenetics. 2015;7:132.

28. Motawi TK, Rizk SM, Ibrahim TM, Ibrahim IA. Circulating microRNAs, miR-92a, miR-100 and miR-143, as non-invasive biomarkers for bladder cancer diagnosis. Cell Biochem Funct. 2016;34(3): $142-148$.

29. Wells GA, Shea B, O'connell D, et al. The Newcastle-Ottawa Scale (NOS) for assessing the quality of nonrandomised studies in metaanalyses. Ottawa, ON: Ottawa Hospital Research Institute; 2000.

30. Shao Y, Geng Y, Gu W, Huang J, Ning Z, Pei H. Prognostic significance of microRNA-375 downregulation in solid tumors: a meta-analysis. Dis Markers. 2014;2014:626185.

31. Tierney JF, Stewart LA, Ghersi D, Burdett S, Sydes MR. Practical methods for incorporating summary time-to-event data into metaanalysis. Trials. 2007;8:16.

32. Begg CB, Mazumdar M. Operating characteristics of a rank correlation test for publication bias. Biometrics. 1994;50(4):1088-1101.

33. Egger M, Smith GD, Schneider M, Minder C. Bias in meta-analysis detected by a simple, graphical test. BMJ. 1997;315(7109):629-634.

34. Rane JK, Erb HH, Nappo G, et al. Inhibition of the glucocorticoid receptor results in an enhanced miR-99a/100-mediated radiation response in stem-like cells from human prostate cancers. Oncotarget. Epub 2016 Jun 21.

35. Leite KR, Tomiyama A, Reis ST, et al. MicroRNA-100 expression is independently related to biochemical recurrence of prostate cancer. J Urol. 2011;185(3):1118-1122. 
36. de Oliveira JC, Scrideli CA, Brassesco MS, et al. Differential miRNA expression in childhood acute lymphoblastic leukemia and association with clinical and biological features. Leuk Res. 2012;36(3):293-298.

37. Li XJ, Luo XQ, Han BW, Duan FT, Wei PP, Chen YQ. MicroRNA100/99a, deregulated in acute lymphoblastic leukaemia, suppress proliferation and promote apoptosis by regulating the FKBP51 and IGF1R/ mTOR signalling pathways. Br J Cancer. 2013;109(8):2189-2198.

38. Bai J, Guo A, Hong Z, Kuai W. Upregulation of microRNA-100 predicts poor prognosis in patients with pediatric acute myeloid leukemia. Onco Targets Ther. 2012;5:213-219.

39. Wang T, Lv M, Shen S, et al. Cell-free microRNA expression profiles in malignant effusion associated with patient survival in non-small cell lung cancer. PLoS One. 2012;7(8):e43268.

40. Zhang B, Zhao R, He Y, et al. MicroRNA 100 sensitizes luminal A breast cancer cells to paclitaxel treatment in part by targeting mTOR. Oncotarget. 2016;7(5):5702-5714.

41. Xu C, Zeng Q, Xu W, et al. miRNA-100 inhibits human bladder urothelial carcinogenesis by directly targeting mTOR. Mol Cancer Ther. 2013;12(2):207-219.

42. Bi Y, Jing Y, Cao Y. Overexpression of miR-100 inhibits growth of osteosarcoma through FGFR3. Tumour Bio. 2015;36(11):8405-8411.

43. Catto JW, Miah S, Owen HC, et al. Distinct microRNA alterations characterize high- and low-grade bladder cancer. Cancer Res. 2009; 69(21):8472-8481.

44. Yang G, Gong Y, Wang Q, Wang Y, Zhang X. The role of miR-100mediated Notch pathway in apoptosis of gastric tumor cells. Cell Signal. 2015;27(6):1087-1101.
45. Klemm F, Bleckmann A, Siam L, et al. $\beta$-catenin-independent WNT signaling in basal-like breast cancer and brain metastasis. Carcinogenesis. 2011;32(3):434-442.

46. Jiang Q, He M, Guan S, et al. MicroRNA-100 suppresses the migration and invasion of breast cancer cells by targeting FZD-8 and inhibiting Wnt/beta-catenin signaling pathway. Tumour Biol. 2016;37(4): 5001-5011.

47. Bhushan L, Kandpal RP. EphB6 receptor modulates micro RNA profile of breast carcinoma cells. PLoS One. 2011;6(7):e22484.

48. Fu HL, Wu de $\mathrm{P}$, Wang XF, et al. Altered miRNA expression is associated with differentiation, invasion, and metastasis of esophageal squamous cell carcinoma (ESCC) in patients from Huaian, China. Cell Biochem Biophys. 2013;67(2):657-668.

49. Xiao F, Bai Y, Chen Z, et al. Downregulation of HOXA1 gene affects small cell lung cancer cell survival and chemoresistance under the regulation of miR-100. Eur J Cancer. 2014;50(8):1541-1554.

50. Chen J, Zheng B, Wang C, et al. Prognostic role of microRNA-100 in various carcinomas: evidence from six studies. Tumour Biol. 2014; 35(4):3067-3071.

51. Chen D, Zhou Y, Du H, Che G. Refute the conclusion made by Jie et al. in "prognostic role of microRNA-100 in various carcinomas: evidence from six studies". Tumour Biol. 2014;35(8):7393-7396.

52. Komatsu S, Ichikawa D, Takeshita H, et al. Prognostic impact of circulating miR-21 and miR-375 in plasma of patients with esophageal squamous cell carcinoma. Expert Opin Biol Ther. 2012;12(Suppl 1): S53-S59.
OncoTargets and Therapy

\section{Publish your work in this journal}

OncoTargets and Therapy is an international, peer-reviewed, open access journal focusing on the pathological basis of all cancers, potential targets for therapy and treatment protocols employed to improve the management of cancer patients. The journal also focuses on the impact of management programs and new therapeutic agents and protocols on

\section{Dovepress}

patient perspectives such as quality of life, adherence and satisfaction The manuscript management system is completely online and includes a very quick and fair peer-review system, which is all easy to use. Visit http://www.dovepress.com/testimonials.php to read real quotes from published authors. 